\title{
Cancer therapy finds a solid target
}

T ost molecular targets for 1 treating cancer prove to be busts. Broadly applicable anticancer therapies have been particularly difficult to develop because of the multiplicity of genetic mutations in a cancer cell: target one mutation and another will slip by, potentially causing the therapy to be ineffective. Recently, however, it has been realized that genetic mutations in cancer often converge on central regulators, namely transcription factors. ${ }^{1}$ New research is now squarely pointing the finger at the oncogene signal transducer and activator of transcription 3 (STAT3) as a promising target for the development of a broad anticancer therapy.

STAT3 is a protein required for gene expression, by which RNA is produced from DNA. Although these transcription factors are often not mutated themselves, their overactivation by other mutations leads to the gene-expression changes that are hallmarks of malignant disease. STAT3 is overactivated at a high frequency in a surprising number of cancers, including leukemias and lymphomas like large granular lymphocyte leukemia and non-Hodgkins lymphoma), as well as solid tumours like breast and prostate cancer. ${ }^{1}$ Furthermore, in multiple myeloma and head and neck cancers, cell growth has been found to require the continual activation of STAT3.

With STAT3 as a central node in the development of many cancers, blocking its overactivation in cancer cells would perhaps preclude having to deal with the multiple protein mutations that can feed into this transcription factor. Early evidence $^{1}$ suggests that this may indeed work, because turning STAT3 off causes some cancer cells to stop growing and even to commit suicide (apoptosis).

Roberto Chiarle and colleagues ${ }^{2}$ have recently demonstrated that STAT3 plays a role in the development of anaplastic large-cell lymphomas, a subset of non-Hodgkins lymphomas. They found that deleting one or both copies of STAT3 in lymphocytes caused lymphoma cells to grow poorly, and that cells still possessing one copy of STAT3 could be made to undergo apoptosis by removing this gene.

Using mice with intact STAT3, the researchers then sought to determine if blocking the activation of STAT3 could have a therapeutic effect. The authors challenged mice with a subcutaneous injection of tumour cells, and 7 days later they treated some of them with STAT3 antisense oligonucleotides that would bind to and inhibit STAT3. In the treated mice no tumour growth was seen, whereas after 14 days the control mice had large tumours. Moreover, giving STAT3 antisense oligonucleotides to mice that already had tumours restricted tumour growth. No adverse effects were observed among the mice that received the oligonucleotides.

Chiarle and colleagues' results, together with other work in the field, ${ }^{1}$ indicate that therapies targeting STAT3 are beneficial. It is therefore of note that other STAT3 inhibitors are under development. One compound, STA-21, has already been shown to inhibit the growth and survival of breast cancer cells. ${ }^{3}$ With STAT3 solidified as a target, further success in the development of a broadly applicable anti-STAT3 therapy may be close at hand. David Secko, Vancouver

\section{References}

1. Yu H, Jove R. The STATs of cancer - new molecular targets come of age [review]. Nat Rev Cancer 2004;4(2): 97-105.

2. Chiarle R, Simmons WJ, Cai H, Dhall G, Zamo A, Raz R, et al. Stat3 is required for ALK-mediated lymphomagenesis and provides a possible therapeutic target. Nat Med 2005;11 (6):623-9.

3. Song H, Wang R, Wang S, Lin J. A low-molecular-weight compound discovered through virtual database screening inhibits Stat 3 function in breast cancer cells. Proc Natl Acad Sci US A 2005;102(13):4700-5. 